Research Paper

\title{
Interaction of CD147 and human epididymis protein 4 promotes invasion and metastasis of ovarian cancer
}

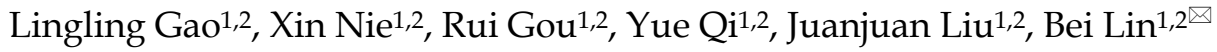 \\ 1. Department of Obstetrics and Gynecology, Shengjing Hospital of China Medical University, No. 36, Sanhao Street, Heping District, Shenyang, 110004, \\ China. \\ 2. Key Laboratory of Maternal-Fetal Medicine of Liaoning Province, Key Laboratory of Obstetrics and Gynecology of Higher Education of Liaoning Province, \\ Liaoning, China. \\ $\square$ Corresponding author: Bei Lin, Department of Obstetrics and Gynaecology, Shengjing Hospital of China Medical University, No.36 Sanhao Street, Heping \\ District, Shenyang, 110004, Liaoning, China. E-mail: linbei88@hotmail.com.
}

(๑) The author(s). This is an open access article distributed under the terms of the Creative Commons Attribution License (https://creativecommons.org/licenses/by/4.0/). See http://ivyspring.com/terms for full terms and conditions.

Received: 2021.05.07; Accepted: 2021.10.18; Published: 2021.10.30

\begin{abstract}
Background: Ovarian cancer is one of the most common malignant tumors in female reproductive system. The expression of CD147 and human epididymis protein 4 (HE4) are both upregulated and associated with malignant progression in ovarian cancer. However, the important role of interaction between CD147 and HE4 in the invasion and metastasis of ovarian cancer remains unclear.

Methods: The co-expression and co-localization of CDI47 and HE4 in cells and tissues of ovarian cancer were detected by co-immunoprecipitation, immunohistochemistry and immunocytochemistry, double-labeling immunofluorescence method. The interaction between CD147 and annexin A2 (ANXA2) was also investigated. Furthermore, we detect the regulatory relationship between CD147 and HE4 by Western blot. Transwell assay and scratch test were conducted to explore the effect of CD147-HE4 interaction on migration and invasion of ovarian cancer.

Results: The protein CD147 and HE4 were co-immunoprecipitated and co-located in the cytoplasm and membrane of ovarian cancer tissues and cells. Both of two proteins were highly expressed and positively associated with advanced FIGO stages, poor differentiation degree and poor prognosis of ovarian cancer, and HE4 was confirmed as an independent risk factor for CD147 in ovarian cancer tissues. What's more, AXNA2 was also identified as a CD147 interacting protein in ovarian cancer. It is further confirmed that interaction between CD147 and HE4 can affect the invasion and metastasis of ovarian cancer.

Conclusion: This study demonstrated that HE4 and ANXA2 were both CD147 interacting proteins, the expression of CDI47 and HE4 can affect each other, and HE4 could promote the invasion and metastasis of ovarian cancer by regulating the expression of $\mathrm{CD} 147$, which may provide novel thought for early diagnosis and therapeutic target of ovarian cancer.
\end{abstract}

Key words: CD147; human epididymis protein 4; ovarian cancer; invasion and metastasis

\section{Introduction}

Ovarian cancer is one of the most common malignancy with the highest mortality rate in gynecological malignant tumors [1]. Due to the concealment of location, lack of specific clinical features and early diagnostic methods, about $70 \%$ of the patients were in the advanced stages at diagnosis, ovarian cancer cells grew rapidly and were prone to invasion, migration and distant metastasis [2]. Although the treatment strategy for ovarian cancer has been greatly improved in recent years, due to the metastasis, recurrence and drug resistance, the 5-year overall survival of patients with ovarian cancer was less than $47 \%$ [3]. So far, many studies have showed varieties of novel biomarkers that can be treated as early diagnosis, prognosis evaluation and monitoring of recurrence of ovarian cancer. However, the development of reliable predictive molecules is urgently required to improve survival for ovarian cancer patients. 
CD147, also known as extracellular matrix metalloproteinase inducer (EMMPRIN) is a singlestranded transmembrane glycoprotein, belonging to the immunoglobulin superfamily (IgSF) [4-5]. The coding gene of CD147 is located at chromosome 19pl3.3, which encodes a total of 268 amino acids (33-66kDa), including signal peptide, extracellular domain, transmembrane domain and intracellular region. The signal peptide region is involved in the biosynthesis and transport of CD147. There are two highly conserved Ig-like domains in the extracellular domain, the transmembrane domain can mediate the interaction between CD147 and membrane proteins. The protein exists in two forms: glycosylated form (HG 40-60 kDa) and core-glycosylated form (LG 32 $\mathrm{kDa})$. CD147, due to different protein distributions and different glycosylation styles, could perform various functions in forms of high and low glycosylation in different tissues, while HG-CD147 is considered as the main functional form [6]. Researches showed that CD147 could be involved in a variety of physiological and pathological processes, including spermatogenesis, embryonic development, tissue and organ formation, wound healing, inflammation, bone reconstruction and so on [7]. At present, large number of studies have confirmed that CD147 was highly expressed in a variety of malignant tumors, and positively correlated with malignant progression of tumors, including breast cancer, hepatocellular carcinoma, gastric cancer, lung cancer, and ovarian cancer [8-12], which suggested that CD147 played a vital role in early diagnosis, prognosis evaluation, invasion and metastasis of various malignancy.

Human epididymal protein 4 (HE4), also called WAP four-disulfide core protein 2 (WFDC2), is an extracellular secretory protein containing two WAP domains, the WFDC2 gene is located on chromosome $20 \mathrm{q} 12-13$ with a total length of about $12 \mathrm{~kb}$, consisting of 5 exons and 4 introns [13]. Because of its specific localization in epididymal epithelial cells, HE4 was initially considered to be a biomarker of epididymal tissue [14]. HE4 was proposed as a novel biomarker for ovarian cancer, and has been approved for clinical detection due to its high specificity and sensitivity in 2003[15]. In recent years, researchers have shown that HE4 was not only expressed in ovarian cancer, but also in other malignant tumors, such as endometrial carcinoma, lung cancer, breast cancer, gastric cancer and pancreatic cancer [16-19], which was closely correlated with the proliferation, invasion and migration of tumors.

We previously showed that CD147 and HE4 were both highly expressed in ovarian cancer tissues [11], and HE4 could interact with ANXA2 to promote malignant biological behavior and EMT process of ovarian cancer [16,20-21]. Some studies have reported that there existed an interaction between CD147 and ANXA2 in hepatocellular carcinoma [22], but there's no evidence whether CD147 could interact with ANXA2 in ovarian cancer. Therefore, in this study, we will explore the interaction between CD147 and HE4 and their relationship with prognosis in ovarian cancer. The function of CD147-HE4 in invasion and metastasis of ovarian cancer was detected by cell function experiments, which will provide a new research direction to explore the interaction between CD147 and HE4 in ovarian cancer.

\section{Methods}

\section{Patients and clinical tissue samples}

A total of 133 ovarian tissues and paraffin-embedded specimens were obtained from Department of Obstetrics and Gynecology in Shengjing Hospital of China Medical University from 2004 to 2013. The pathological diagnosis of all samples was confirmed by in-house experts, as follows: malignant group, $\mathrm{n}=96$; borderline group, $\mathrm{n}=11$; benign group, $\mathrm{n}=14$; normal group, $\mathrm{n}=12$ (Table 1 ). All cases were primary epithelial ovarian cancer patients with complete clinical and pathological data, which were diagnosed by pathologists at the Shengjing Hospital of China Medical University, and patients with chemotherapy, radiotherapy, and hormone therapy before surgery were not included in this study (clinic-pathological parameters seen in Table 2). Patients in the malignant tumor group were 19-83 years of age (average: 55.21 years), those in the borderline tumor group were 26-84 years of age (average: 49.40 years), patients in the benign tumor group were 28-79 years of age (average: 55 years), and patients in the normal ovarian group were 36-57 (average: 44.17 years). No significant difference was found among the ages of each group $(P>0.05)$. The pathological types of ovarian cancer were as follows: serous adenocarcinoma $(n=52)$, mucinous adenocarcinoma $(\mathrm{n}=7)$, endometrioid carcinoma $(n=10)$, clear cell carcinoma $(n=8)$ and other pathological types (adenocarcinoma) $(n=19)$. In malignant ovarian tumor group, there were 11, 28, 57 cases of well, moderate, and poor differentiations, respectively. According to the criteria of the International Federation of Gynecology and Obstetrics (FIGO, 2009), each clinical surgical pathological stage was judged as follows: FIGO stages I-II (39 cases) and FIGO stages III-IV (57 cases). Lymph node metastasis was judged as follows: no metastasis (57 cases), metastasis (20 cases), and no lymphadenectomy (19 cases). This study was 
approved by the Ethics Committee of Shengjing hospital of China Medical University.

\section{Immunohistochemistry and Immunocyto- chemistry staining}

Consecutive sections (5 $\mu \mathrm{m}$ thick) were processed from the ovarian tissue specimens fixed with $10 \%$ formalin and embedded in paraffin. The expressions of CD147 and HE4 in ovarian tissues were detected by streptavidin-peroxidase (SP) staining. Positive and negative controls were routinely used. The working concentrations of primary antibodies against CD147 and HE4 used were 1:150 (Proteintech, Wuhan, China, Cat\# 11989-1-AP) and 1:50 (Abcam, Cambridge, UK, Cat\# ab200828) respectively. The staining procedure was performed based on the manufacturer's instructions. The presence of buffy granules in the cell membrane and cytoplasm were regarded as positive. According to the chromatosis intensity, no pigmentation, light yellow, brown yellow, and dark brown are scored 0, 1, 2, and 3, respectively. The percentage of pigmented cells in the whole section was as follows: $<5 \% \%$ are $0,5 \%-25 \%$ : $1,26 \%-50 \%: 2,51 \%-75 \%: 3$, and $>75 \%$ : 4 . The final score was obtained by multiplying the two scores above: $0-2$ scores (-); 3-4 scores (+); 5-8 scores (++); and 9-12 scores (+++). Two pathologists who had no knowledge of the patient's data examined the sections independently to control error.

When cells grow at exponential phase, $0.25 \%$ trypsin were used to digest the cells, and mixed with culture medium containing 10\% FBS to prepare single-cell suspension. When adherent cells grow in a single layer with $30-40 \%$ confluence, cells were washed with cold PBS three times, and then fixed with $4 \%$ paraformaldehyde for $20 \mathrm{~min}$. The following procedures were conducted the same as immunohistochemistry. The working concentrations of primary antibodies against CD147 and HE4 were 1:100 (Proteintech, Wuhan, China, Cat\# 11989-1-AP) and 1:50 (Abcam, Cambridge, UK, Cat\# ab109298), respectively. PBS was used to replace the primary antibody for negative control, and secondary antibody alone was also used as a negative control.

\section{Cell culture and transfection}

All ovarian cancer cell lines (CAOV3, SKOV3, OVCAR3, and ES-2) were obtained from the Institute of Biochemistry and Cell Biology, Chinese Academy of Sciences (Shanghai, China). CaoV3 and OVCAR3 cells were cultured routinely in RPMI 1640 culture medium with $10 \%$ fetal bovine serum (FBS). ES-2 and SKOV3 were propagated in McCoy's 5A with $10 \%$ FBS. All cell lines were grown at $37^{\circ} \mathrm{C}$ in a humidified atmosphere with $5 \% \mathrm{CO} 2$.
OVCAR3 and ES-2 cells were employed to construct the stably low CD147 expression cells: OVCD147-L group and negative control group (OV-NC) and ES-CD-147-L group and negative control group (ES-NC). The lentivirus-mediated CD147 RNAi sequence is: 5'-GUUCUUCGUGAGUUCCUCTT-3'. OVCAR3 and ES-2 cells were transfected with HE4 siRNA (GenePharma, Shanghai, China) using the Lipofectamine 3000 Transfection Kit (Invitrogen, USA) according to the manufacturer's instructions. HE4 siRNA sequences were as follows: siHE4-1: sense: 5'-ACCAGAACUGCACGCAAGATT-3'; antisense: 5'-UCUUGCGUGCAGUUCUGGUT-3'; siHE4-2: sense: 5'-AGGUGAACAUUAACUUU CTT-3'; antisense: 5'-GGAAAGUUAAUGUUCACC UTT-3'. The following cells of stably low ANXA2 expression were constructed: OV-shA2-1/OV-shA2-2 cells and negative control cells (OV-NC) and ES-shA2-1/ES-shA2-2 and negative control cells (ES-NC). The lentivirus-mediated ANXA2 shRNA (Hanbio Biotechnology, Shanghai, China) sequences are: GCTCTGTCATTGATTATGAACTGAT for ANXA2-shRNA1, TGGAGTGAAGAGGAAAGGAA CTGAT for ANXA2-shRNA2. The interference effect was detected by Western blot.

\section{Double-labeling immunofluorescence}

The double-labeling immunofluorescence was conducted to detect the co-expression and co-location of CD147 and HE4 proteins. Ovarian cancer tissues and cells were simultaneously incubated with primary antibodies against CD147 (1:100 [mouse], Santa Cruz, CA, USA, Cat\# sc-21746) and HE4 (1:100 [rabbit], Abcam, Cambridge, UK, Cat\# ab109298). The primary antibody was replaced by PBS for negative controls. The working concentrations of tetraethyl rhodamineisothiocyanate (TRITC) and fluorescein isothiocyanate (FITC) were 1:500. 4,6-diamidino-2phenylindole (DAPI) was used to stain the nuclei. The following procedure was conducted according to the manufacturer's instructions.

\section{Co-immunoprecipitation and Western Blot}

The pre-cooled RIPA buffer (500ul) was added to the precipitation of ovarian cancer cells OVCAR3 and ES-2, then incubated at $4{ }^{\circ} \mathrm{C}$ for 30 minutes. After centrifugated at $12000 \mathrm{~g}$ for 30 minutes at $4{ }^{\circ} \mathrm{C}$, the supernatant was collected and then added $2 \mu \mathrm{g}$ mouse anti-CD147 monoclonal antibody (Santa Cruz, CA, USA) and 2 ug rabbit anti-HE4 monoclonal antibody (Abcam), and relative negative control mouse or rabbit IgG antibody (BIOSS, China) were added, respectively, followed by incubation overnight at 4 ${ }^{\circ} \mathrm{C} .40 \mu \mathrm{L}$ protein A/G-agarose bead (Santa Cruz, CA, USA) were added the next day and agitated at $4^{\circ} \mathrm{C}$ for 
4h. All samples were then centrifuged at 2,500 $\mathrm{g}$ for 5 $\min$ at $4^{\circ} \mathrm{C}$ and washed with lysis buffer three times to collect the precipitation. The immunoprecipitation was analyzed by $10 \%$ SDS gel electrophoresis, and rabbit anti-HE4 monoclonal antibody (Abcam) and rabbit anti-CD147 polyclonal antibody (Proteintech) were used for western blot analysis. Proteins were detected with the Immobilon ${ }^{\circledR}$ western chemiluminescent horseradish peroxidase substrate (Millipore, Billerica, MA, USA). The experiments were repeated three times.

\section{Wound healing assay}

Wound healing assay was conducted as the previous study, cells were seeded in a six-well plate. When cell confluence reached $90 \%$, a $100-\mu \mathrm{L}$ micropipette tip was applied to scratch a wound gently. The cells were washed three times with PBS and incubated with serum-free medium for $48 \mathrm{~h}$. The migration distance was observed under a microscope. The experiment was repeated three times.

\section{Transwell assay}

Transwell assay was performed as the previous study. The upper Transwell chamber (Corning, Inc., Corning, NY, USA) was covered with 70uL basement membrane Matrigel solution (Corning, Bedford, MA, USA, Cat\# 354234) and incubated overnight at $37^{\circ} \mathrm{C}$. A total of $2 \times 10^{4}$ cells $/ 200 \mu \mathrm{L}$ were seeded in the upper chamber with serum-free medium, and the lower chamber was filled with $500 \mu \mathrm{L}$ of medium supplemented with $10 \%$ FBS. After $48 \mathrm{~h}$ of conventional incubation at $37{ }^{\circ} \mathrm{C}$, the Transwell chambers were washed three times with PBS, then cells were fixed with $4 \%$ paraformaldehyde for $30 \mathrm{~min}$ and stained with crystal violet for $30 \mathrm{~min}$, the Matrigel and cells from the inner surface of the chamber were gently removed with a cotton swab. The number of transmembrane cells was observed and counted under a microscope. The experiment was repeated three times.

\section{Statistical analysis}

Statistical analyses were performed by SPSS21.0 software (IBM Corporation, Armonk, NY, USA), the data results were expressed as mean $\pm \mathrm{SD}$. Difference between two groups were calculated by student's t-test and the Chi-squared, and one-way ANOVA was used for comparison among more than two groups. The survival curve was measured by Kaplan-Meier and Log-rank test, and the correlation between the two proteins was analyzed by Spearman analysis and Regression model. Bilateral test $P<0.05$ was regarded as statistically significant. ${ }^{*} P<0.05,{ }^{* *} P<0.01$, and *** $P<0.001$.

\section{Results}

\section{Co-expression of CD147 and HE4 in ovarian cancer cells and tissues}

The expressions of CD147 and HE4 protein in ovarian cancer cell lines OVCAR3 and ES-2 were detected by Western blot, immunocytochemistry and double-label immunofluorescence assays (Figure 1A and Figure 2A). We further confirmed the expression of CD147 and HE4 protein in different ovarian tissues with immunohistochemistry (Figure 3A). Interaction between CD147 and HE4 was detected by co-immunoprecipitation in OVCAR3 and ES-2 cells (Figure 1B-C). Double-label immunofluorescence assays showed that CD147 protein labeled by green fluorescence and HE4 protein labeled by red fluorescence were observed in the cell membrane and cytoplasm of OVCAR3 and ES-2 cells (Figure 2B) and ovarian cancer tissues (Figure 3B), and the co-localization sites of CD147 and HE4 were observed by the overlapping orange fluorescence. In addition, we also detected that CD147 can also interacted with ANXA2 in both OVCAR3 and ES-2 cells (Supplementary Figure 1A-B).

\section{Expression and co-localization of CDI47 and HE4 in different ovarian tissues}

Immunohistochemistry showed that CD147 was mainly expressed in the cell membrane and cytoplasm. The positive expression rate of CD147 in malignant group was $90.6 \%$ (87/96), which was significantly higher than that in borderline group (63.6\%, [7/11]), benign group $(42.9 \%,[6 / 14])$, and normal ovarian tissues $(33.3 \%,[4 / 12])$ (all $P<0.05)$. What's more, the positive expression rates of CD147 in borderline group and benign group were also higher than that in normal tissues, but the difference was not statistically significant (both $P>0.05$ ). According to the expression of CD147, 96 cases of ovarian cancer were divided into low $(-/+)$ and high $(++/+++)$ CD147 expression group, the results suggested that the high positive expression rate of CD147 in malignant group was 58.3\% (56/96), which was higher than that in benign group $(14.3 \%,[2 / 14])$ and normal tissues $(8.3 \%,[1 / 12])$ (both $P<0$. 05) (Table 1, Figure 3A).

HE4 was also mainly located in cell membrane and cytoplasm. The positive expression rate of HE4 in malignant group $(86.5 \%$, [83/96]) was higher than that in borderline group $(54.6 \%,[6 / 11])$, benign group $(50 \%,[7 / 14])$ and normal tissues (33.3\%, [4/12]) (all $P<0.05)$. Moreover, the positive expression rate of HE4 in borderline group and benign group was higher than that in normal tissues, but the difference was not statistically significant (both $P>0$. 05). We further 
divided malignant tumors into low $(-/+)$ and high $(++/+++)$ HE4 expression group, the results showed that the high positive expression rates of HE4 in malignant group, borderline group, benign group and normal group were $67.7 \%$ (65/96), 36.4\% (4/11),

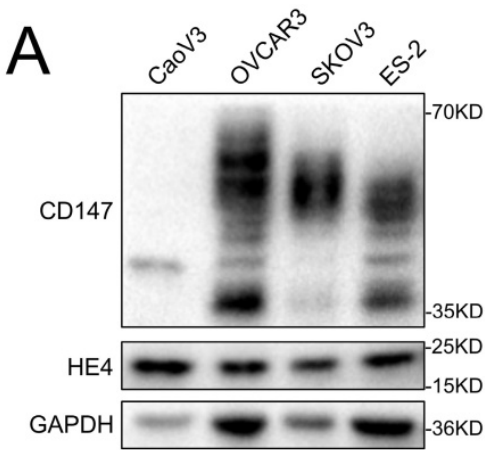

B
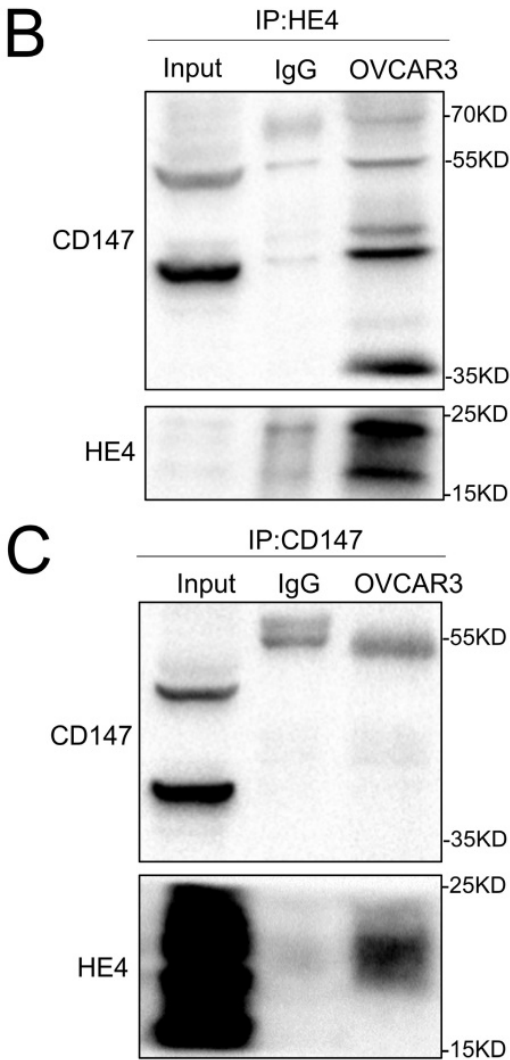

$28.6 \%(4 / 14)$ and $16.7 \%(2 / 12)$, respectively. The high positive expression rate in malignant group was significantly higher than that in benign group and normal tissues (both $P<0.05$ ) (Table 1, Figure 3A).
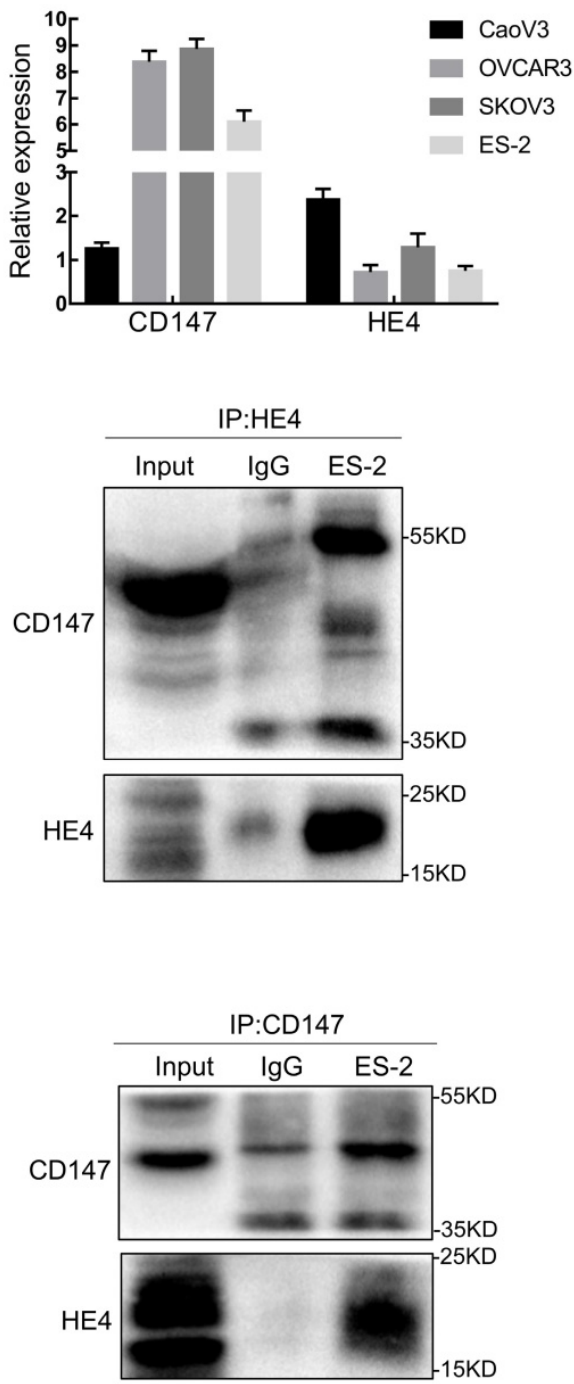

Figure 1. Expression and co-immunoprecipitation of CD147 and HE4 in ovarian cancer cells. (A) The expression of CD147 and HE4 in ovarian cancer cell lines (CaoV3, OVCAR3, SKOV3 and ES-2) detected by Western blot. (B) Cell lysates from OVCAR3 and ES-2 cells were subjected to immunoprecipitation with anti-HE4 antibody and immunoblotted with anti-CD147 antibody. (C) Cell lysates from OVCAR3 and ES-2 cells was immunoprecipitated with anti-CD147 antibody and immunoblotted with anti-HE4 antibody, "IgG" represents the negative control. "Input" indicates total cell lysate of OVCAR3 and ES-2.

Table 1. Expression of CD147 and HE4 in different ovarian tissues

\begin{tabular}{|c|c|c|c|c|c|c|c|c|c|c|c|c|c|}
\hline \multirow[t]{2}{*}{ Groups } & \multirow[t]{2}{*}{ Cases } & \multicolumn{6}{|c|}{ CD147 } & \multicolumn{6}{|c|}{$\mathrm{HE} 4$} \\
\hline & & - & + & ++ & +++ & Positive (\%) & High positive (\%) & - & + & ++ & +++ & Positive (\%) & High positive (\%) \\
\hline Malignant & 96 & 9 & 31 & 32 & 24 & $90.6 \mathrm{a}, \mathrm{b}, \mathrm{c}$ & $58.3 \mathrm{~d}, \mathrm{e}$ & 13 & 18 & 19 & 46 & 86.5 e,f,g & $67.7 \mathrm{~h}, \mathrm{j}$ \\
\hline Borderline & 11 & 4 & 4 & 2 & 1 & 63.6 & 27.3 & 5 & 2 & 2 & 2 & 54.5 & 36.4 \\
\hline Benign & 14 & 8 & 4 & 2 & 0 & 42.9 & 14.3 & 7 & 3 & 2 & 2 & 50.0 & 28.6 \\
\hline Normal & 12 & 8 & 3 & 1 & 0 & 33.3 & 8.3 & 8 & 2 & 1 & 1 & 33.3 & 16.7 \\
\hline
\end{tabular}

Note: a,b,c indicated that the positive expression rate of CD147 in malignant tissues was compared with that in borderline group, benign group and normal tissues, all $P<0.05$ $\left(P_{\mathrm{a}}=0.035, P_{\mathrm{b}}<0.001, P_{\mathrm{c}}<0.001\right)$ d,e; indicated that the high positive rate of CD147 in malignant tissues is higher than that in benign group and normal tissues, all $P<0.05$

$\left(P_{\mathrm{d}}=0.002, P_{\mathrm{e}}=001\right)$; e,f,g indicated that the positive expression rate of HE4 in malignant tissues was compared with that in borderline group, benign group and normal tissues, all $P<0.05\left(P_{\mathrm{e}}=0.024, P_{\mathrm{f}}=0.003, P_{\mathrm{g}}<0.001\right)^{\mathrm{h},}$; indicated that the high positive rate of HE4 in malignant tissues is higher than that in benign group and normal tissues, both $P<0.05\left(P_{\mathrm{h}}=0.005, P_{\mathrm{j}}=002\right)$. 

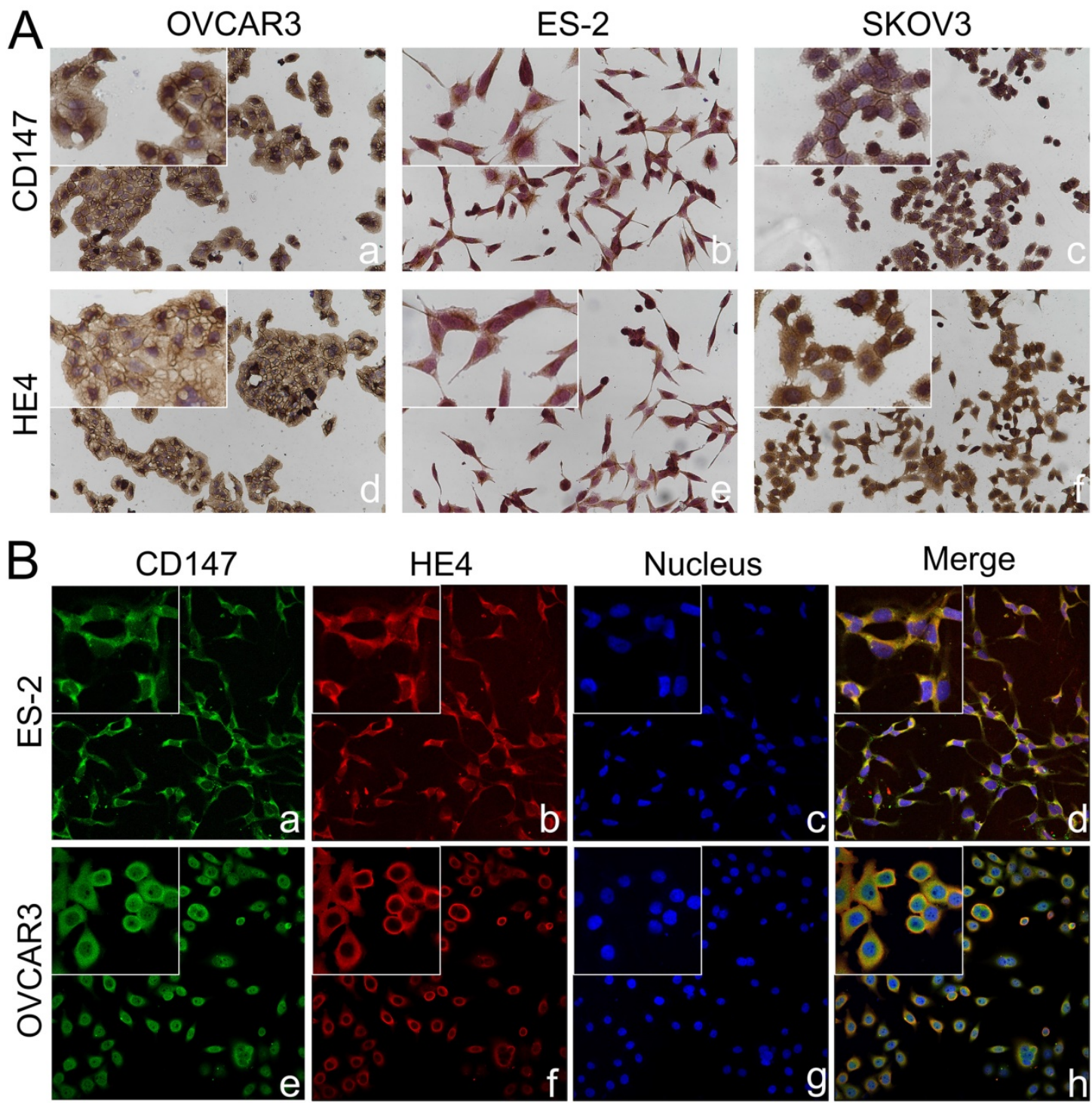

Figure 2. Co-localization of CDI47 and HE4 in ovarian cancer cells. (A) Expression of CD147 and HE4 in ovarian cancer cell lines OVCAR3 (a, d), ES-2 (b, e), SKOV3 (c, f) conducted by immunocytochemistry. (B) The co-localization of CD147 (green) and HE4 (red) in OVCAR3 (a-d) and ES-2 cells (e-h) analyzed by double-labeling immunofluorescence assays, "blue" represents nucleus; "orange" represents the co-localization of CD147 and HE4. The small box at the top-left corner was magnified by $400 \times$ $(\mathrm{A}, \mathrm{B})$.

\section{Relationships between the expression of CD147, HE4 and clinicopathological parameters of ovarian cancer}

The positive expression rate of CD147 in stages III-IV of ovarian cancer was $96.5 \%(55 / 57)$, which was higher than that in stages I-II $(82.1 \%,[32 / 39])(P<0.05)$ (Table 2, Figure 4A). The positive expression rate of CD147 in the poor differentiation group $(96.5 \%$, [55/57]) was significantly higher than that in well differentiation group $(63.6 \%$, [7/11]) $(P<0.05)$ (Table 2, Figure 4B). The positive expression rate of CD147 in lymphnode metastasis group was 95\% (19/20), which was higher than that in non-metastasis group $(87.7 \%$, [50/57]), but no statistical significance $(P>0.05) .96$ cases of epithelial ovarian malignant tumors were further divided into low $(-/+)$ and high $(++/+++)$
CD147 expression group based on the CD147 expression in ovarian cancer. The results indicated that the high positive expression rate of CD147 in III-IV stages $(66.7 \%,[38 / 57])$ was higher than that in stages I-II $(46.2 \%,[18 / 39]) \quad(P<0.05)$. The high expression rate of CD147 gradually decreased as the degree of differentiation increased, and the high positive expression rate in low differentiation group (70.2\% [40/57]) was significantly higher than that in well differentiated group $(18.2 \%,[2 / 11])(P<0.05)$. Although the high expression rate in the low differentiation group was also higher than that in the moderate differentiation group, the difference was not statistically significant. No significant difference in the expression of CD147 with respect to age at diagnosis and pathological type $(P>0.05)$ (Table 2$)$. 


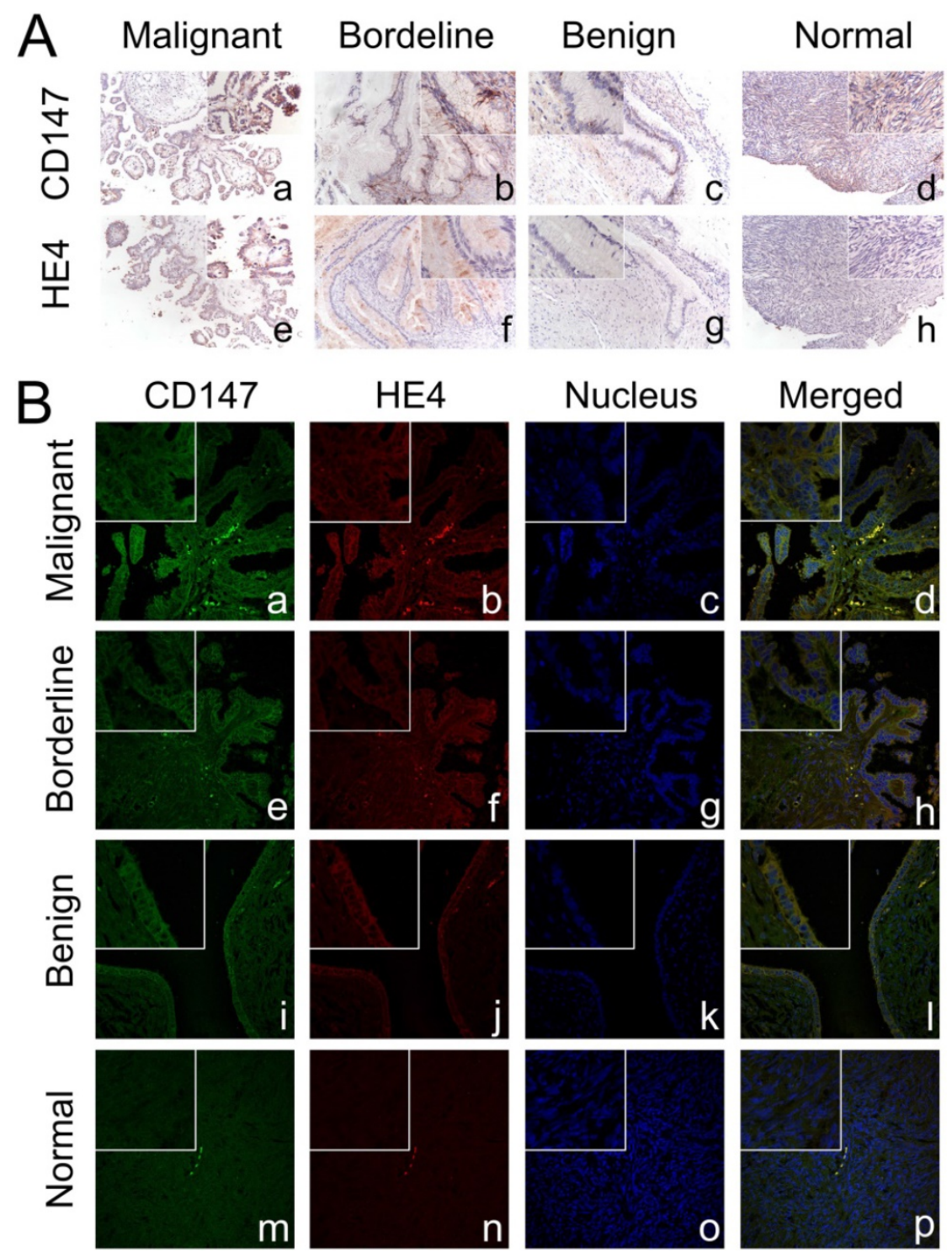

Figure 3. Expression and co-localization of CD147 and HE4 in different ovarian tissues. (A) The expression of CD147 and HE4 in malignant group (a, e), borderline group (b, f), benign group (c, g), and normal ovarian tissues (d, h) detected by immunohistochemistry. (B) Co-localization of CD147 and HE4 analysed by double-labeling immunofluorescence assay in malignant group (a-d), borderline group (e-h), benign group (i-l), and normal tissues (m-p). The color of "green" indicates CD147; "red" indicates HE4; "blue" indicates nucleus; "orange" indicates the co-localization of CD147 and HE4. The small box at the top-left corner was magnified by $400 \times$ (A, B).
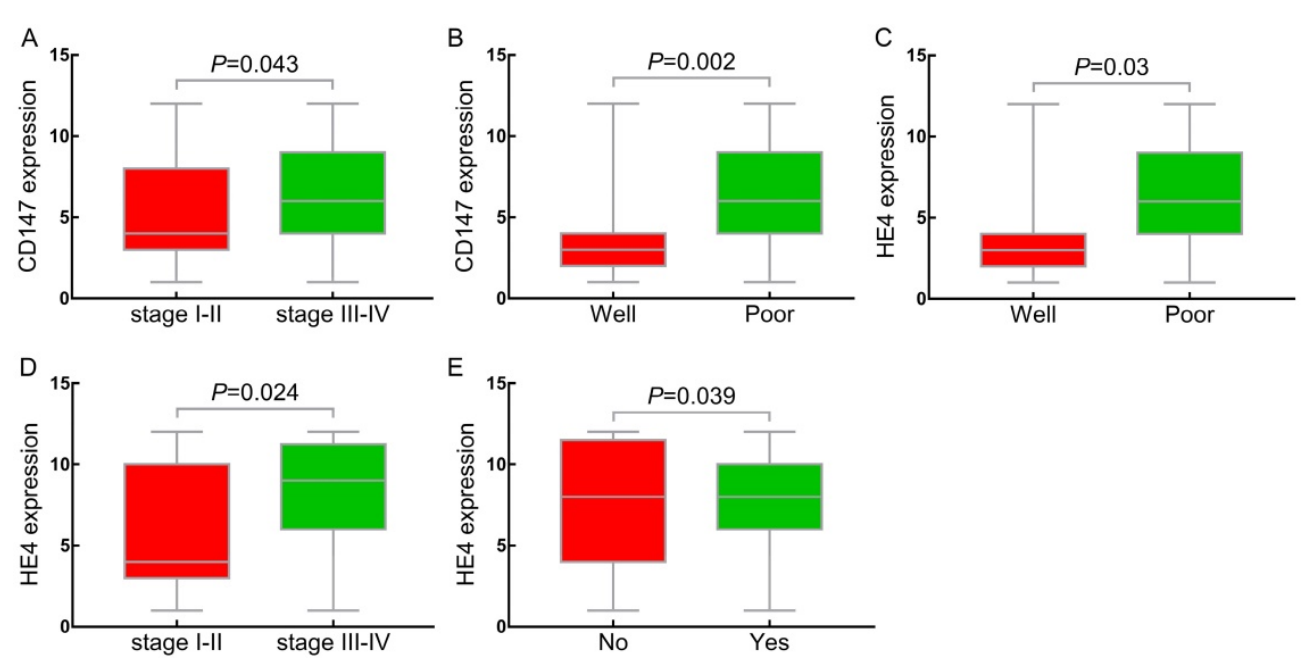

Figure 4. Relationship between CD147, HE4 expression and clinicopathologic parameters. (A, D) Compared the expression of CDI47, HE4 protein in FIGO stages (I-II) with stages (III-IV) in OC, respectively; (B, C) Compared expression of CD147, HE4 protein in well differentiation group with poor differentiation group in OC, respectively. (E) Compared expression of HE4 protein in lymph node metastasis with non-metastasis group in OC. OC: ovarian cancer. 
Table 2. Relationships between the expression of CD147, HE4 and clinicopathological parameters of 96 ovarian cancer patients

\begin{tabular}{|c|c|c|c|c|c|c|c|c|c|}
\hline \multirow[t]{2}{*}{ Groups } & \multirow[t]{2}{*}{ Cases } & \multicolumn{4}{|l|}{ CD147 } & \multicolumn{4}{|l|}{ HE4 } \\
\hline & & Positive rate (\%) & $P$-value & High expression rate (\%) & $P$-value & Positive rate (\%) & $P$-value & High expression rate (\%) & $P$-value \\
\hline \multicolumn{10}{|l|}{ Age at diagnosis } \\
\hline$<59$ & 58 & $52 / 58(89.7 \%)$ & \multirow[t]{2}{*}{$P>0.05$} & $33 / 58(56.9 \%)$ & \multirow[t]{2}{*}{$P>0.05$} & $49 / 58(84.5 \%)$ & \multirow[t]{2}{*}{$P>0.05$} & $41 / 58(70.7 \%)$ & \multirow[t]{2}{*}{$P>0.05$} \\
\hline$\geq 59$ & 38 & $35 / 38(92.1 \%)$ & & $23 / 38(60.5 \%)$ & & $34 / 38(89.5 \%)$ & & $24 / 38(63.2 \%)$ & \\
\hline \multicolumn{10}{|l|}{ Pathological type } \\
\hline Serous & 52 & $47 / 52(90.4 \%)$ & \multirow[t]{5}{*}{$P>0.05$} & $31 / 52(59.6 \%)$ & \multirow[t]{5}{*}{$P>0.05$} & $48 / 52(88.5 \%)$ & \multirow[t]{5}{*}{$P>0.05$} & $35 / 52(67.3 \%)$ & \multirow[t]{5}{*}{$P>0.05$} \\
\hline Mucious & 7 & $4 / 7(57.1 \%)$ & & $1 / 7(14.3 \%)$ & & $4 / 7(57.1 \%)$ & & $3 / 7(42.9 \%)$ & \\
\hline Endometrioid & 10 & 9/10 (90\%) & & $8 / 10(80 \%)$ & & 9/10 (90\%) & & $8 / 10(80 \%)$ & \\
\hline Clear cell carcinoma & 8 & $8 / 8(100 \%)$ & & $7 / 8(87.5 \%)$ & & $7 / 8(87.5 \%)$ & & $7 / 8(87.5 \%)$ & \\
\hline $\begin{array}{l}\text { Poorly differentiated } \\
\text { adenocarcinoma }\end{array}$ & 19 & $19 / 19(100 \%)$ & & $9 / 19(47.4 \%)$ & & $17 / 19(89.5 \%)$ & & $12 / 19(63.2 \%)$ & \\
\hline \multicolumn{10}{|l|}{ FIGO stage } \\
\hline I-II & 39 & $32 / 39(82.1 \%)$ & \multirow[t]{2}{*}{$P=0.043$} & $18 / 39(46.2 \%)$ & \multirow[t]{2}{*}{$P=0.045$} & $30 / 39(76.9 \%)$ & \multirow[t]{2}{*}{$P=0.024$} & $19 / 39(48.7 \%)$ & \multirow[t]{2}{*}{$P=0.001$} \\
\hline III-IV & 57 & $55 / 57(96.5 \%)$ & & $38 / 57(66.7 \%)$ & & $53 / 57(93 \%)$ & & $46 / 57(80.7 \%)$ & \\
\hline \multicolumn{10}{|l|}{ Differentiation } \\
\hline Well & 11 & $7 / 11(63.6 \%)$ & \multirow{3}{*}{$\begin{array}{l}\text { Pwell. vs } \\
\text { poor } \\
=0.002\end{array}$} & $2 / 11(18.2 \%)$ & \multirow{3}{*}{$\begin{array}{l}\text { Pwell. } \\
\text { vs poor } \\
=0.001\end{array}$} & $7 / 11(63.6 \%)$ & \multirow{3}{*}{$\begin{array}{l}\text { Pwell. vs } \\
\text { poor } \\
=0.030\end{array}$} & $3 / 11(27.3 \%)$ & \multirow{3}{*}{$\begin{array}{l}\text { Pwell. vs } \\
\text { poor } \\
=0.002\end{array}$} \\
\hline Moderate & 28 & $25 / 28(89.3 \%)$ & & $14 / 28(50 \%)$ & & $24 / 28(85.7 \%)$ & & $19 / 28(67.9 \%)$ & \\
\hline Poor & 57 & $55 / 57(96.5 \%)$ & & $40 / 57(70.2 \%)$ & & $52 / 57(91.2 \%)$ & & $43 / 57(75.4 \%)$ & \\
\hline \multicolumn{10}{|c|}{ Lymphnode metastasis } \\
\hline No & 57 & $50 / 57(87.7 \%)$ & \multirow[t]{3}{*}{$P>0.05$} & $30 / 57(52.6 \%)$ & \multirow[t]{3}{*}{$P>0.05$} & $47 / 57(82.5 \%)$ & \multirow[t]{3}{*}{$P>0.05$} & $34 / 57(59.6 \%)$ & \multirow[t]{3}{*}{$P=0.039$} \\
\hline Yes & 20 & $19 / 20(95 \%)$ & & $14 / 20(70 \%)$ & & $19 / 20(95 \%)$ & & $17 / 19(85 \%)$ & \\
\hline Unknown & 19 & $18 / 19(94.7 \%)$ & & $12 / 19(63.2 \%)$ & & $17 / 19(89.5 \%)$ & & $14 / 19(73.7 \%)$ & \\
\hline
\end{tabular}

In accordance with CD147, the positive expression rate of HE4 in III-IV stages (93\%, [53/57]) was significantly higher than that in stages I-II $(76.9 \%$, [30/39]) $(P<0.05)$ (Table 2, Figure 4C). The positive expression rate of HE4 in poor differentiation group was $91.2 \%$ (52/57), which was significantly higher than that in high differentiation group $(63.6 \%,[7 / 11])$ $(P<0.05)$ (Table 2, Figure 4D). Similarly, we further divided the ovarian epithelial malignant tumor group into HE4 low $(-/+)$ and high $(++/+++)$ expression group. The results showed that the high positive expression rate of HE4 in stages III IV (80.7\%, [46/57]) was significantly higher than that in stages I $\sim$ II $(48.7 \%$, [19/39]) $(P<0.05)$. With the decrease of differentiation degree, the expression rate of HE4 increased gradually, and high positive expression rate in low differentiation group $(75.4 \%,[43 / 57])$ was significantly higher than that in high differentiation group $(27.3 \%,[3 / 11])(P<0.05)$. The high expression rate of HE4 in lymph node metastasis group was $85 \%$ $(17 / 19)$, which was significantly higher than that in non-metastasis group $(59.6 \%,[34 / 57]) \quad(P<0.05)$ (Figure 4E). No significant difference was showed in the expression of HE4 with age at diagnosis and pathological type $(P>0.05)$ (Table 2$)$.

\section{CD147 and HE4 overexpression associated with poor prognosis in ovarian cancer}

A total of 96 cases of ovarian cancer patients were followed up until January 1, 2018. Among these ovarian cancer patients, 14 patients were lost follow-up and 40 died. The longest and shortest survival time were 119 and 1 months, respectively. The mortality rates of patients with high expression of CD147 and HE4 were 52.9\% (29/56) and 50.8\%
(33/65) in epithelial ovarian malignant tumors, respectively, which were significantly higher than that in CD147 low expression group (27.5\%, [11/40]) and HE4 low expression group (22.6\%, [7/31]). Kaplan-Meier analysis showed that the average survival time in CD147 and HE4 high expression group were 45.73 and 44.92 months, respectively. While the average survival time in CD147 and HE4 low expression group were 52.65 and 56.35 months, respectively. The high expression of CD147 and HE4 was significantly associated with a shortened overall survival in patients with ovarian cancer $(P=0.018$ and $P=0.005$, respectively) (Figure 5A-B). Furthermore, FIGO stages (I-II vs III-IV) and lymph node metastasis (No vs Yes) were also associated with poor prognosis in ovarian cancer (all $P<0.05)$ (Supplementary Table 1, Figure 5C-D).

Cox regression model was adopted to analyze the clinicopathological parameters affecting the prognosis of patients with ovarian cancer. Univariate analysis showed FIGO stages $(\mathrm{HR}=3.132,95 \%$ $\mathrm{CI}=1.430-6.858, \quad P=0.004), \quad$ lymphnode metastasis $(\mathrm{HR}=2.82, \quad 95 \% \quad \mathrm{CI}=1.328-5.990, \quad P=0.007), \quad \mathrm{CD} 147$ expression level (HR=2.158, 95\% CI=1.044-4.460, $P=0.045)$ and HE4 expression level $(\mathrm{HR}=2.492$, 95\% $\mathrm{CI}=1.093-5.680, P=0.03$ ) were significantly correlated with overall survival time (Table 4). Multivariate analysis showed that CD147 expression level $(\mathrm{HR}=3.126,95 \% \mathrm{CI}=1.025-9.530, P=0.004)$ and lymph node metastasis $(\mathrm{HR}=2.818,95 \% \mathrm{CI}=1.072-7.408$, $P=0.036)$ were independent risk factors affecting the prognosis of ovarian cancer. The univariate and multivariate Cox regression analysis were further visualized by forest maps (Figure 6). 
A
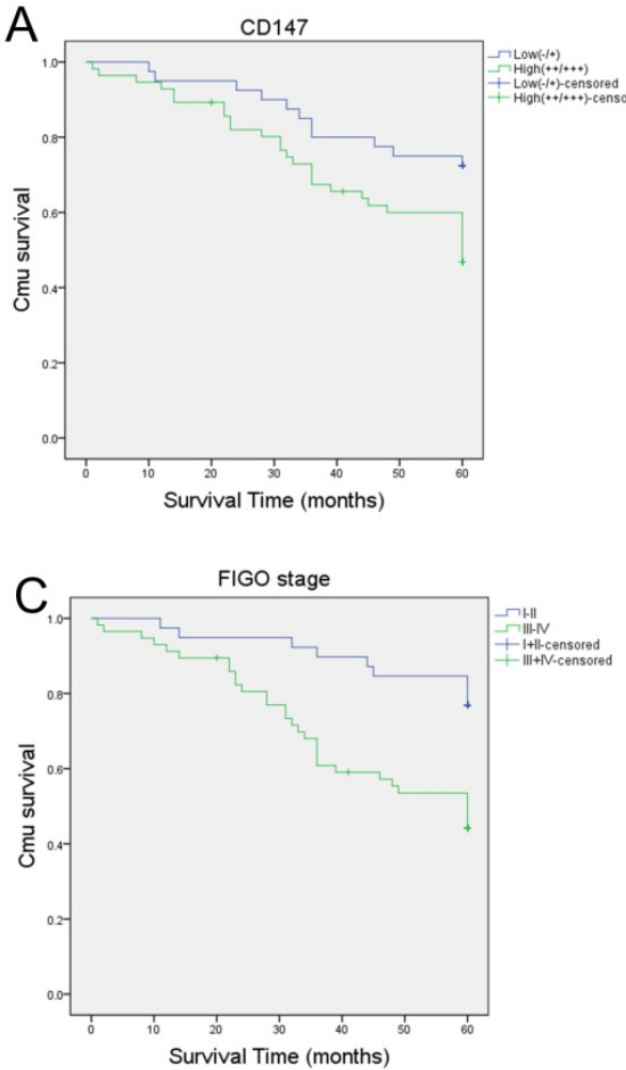

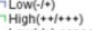
- Low $(-+*)$-censored
High $(*+*+*+)$-censored
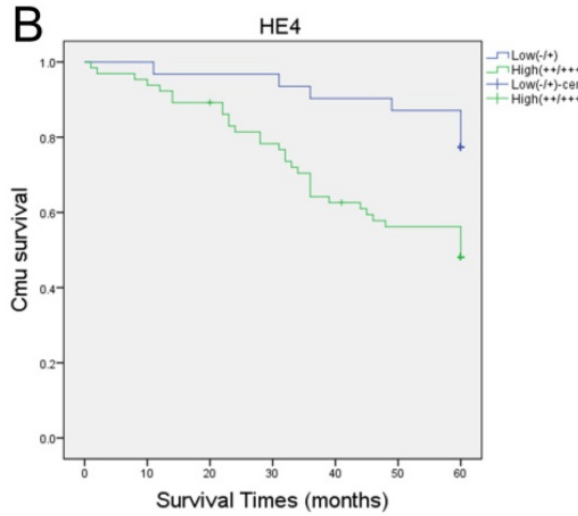

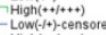
- Low $(-1+$ - censored
- High $(*++*+*)$-censored

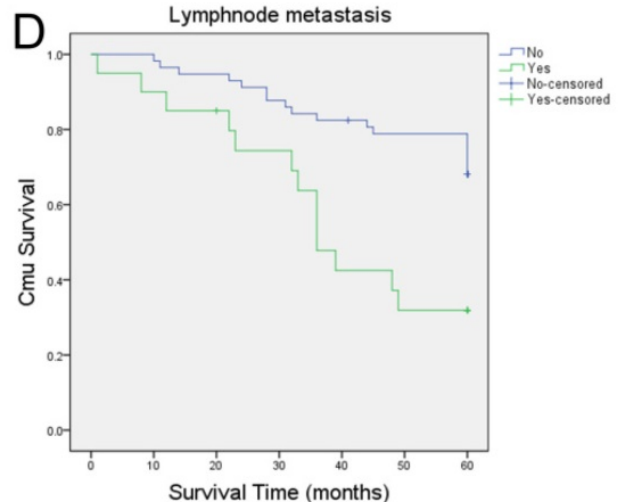

Figure 5. Kaplan-Meier analysis of the prognosis of ovarian cancer. Relationship between prognosis and high or low CD147 expression (A), high or low HE4 expression (B), FIGO stages (C), lymphnode metastasis (D).

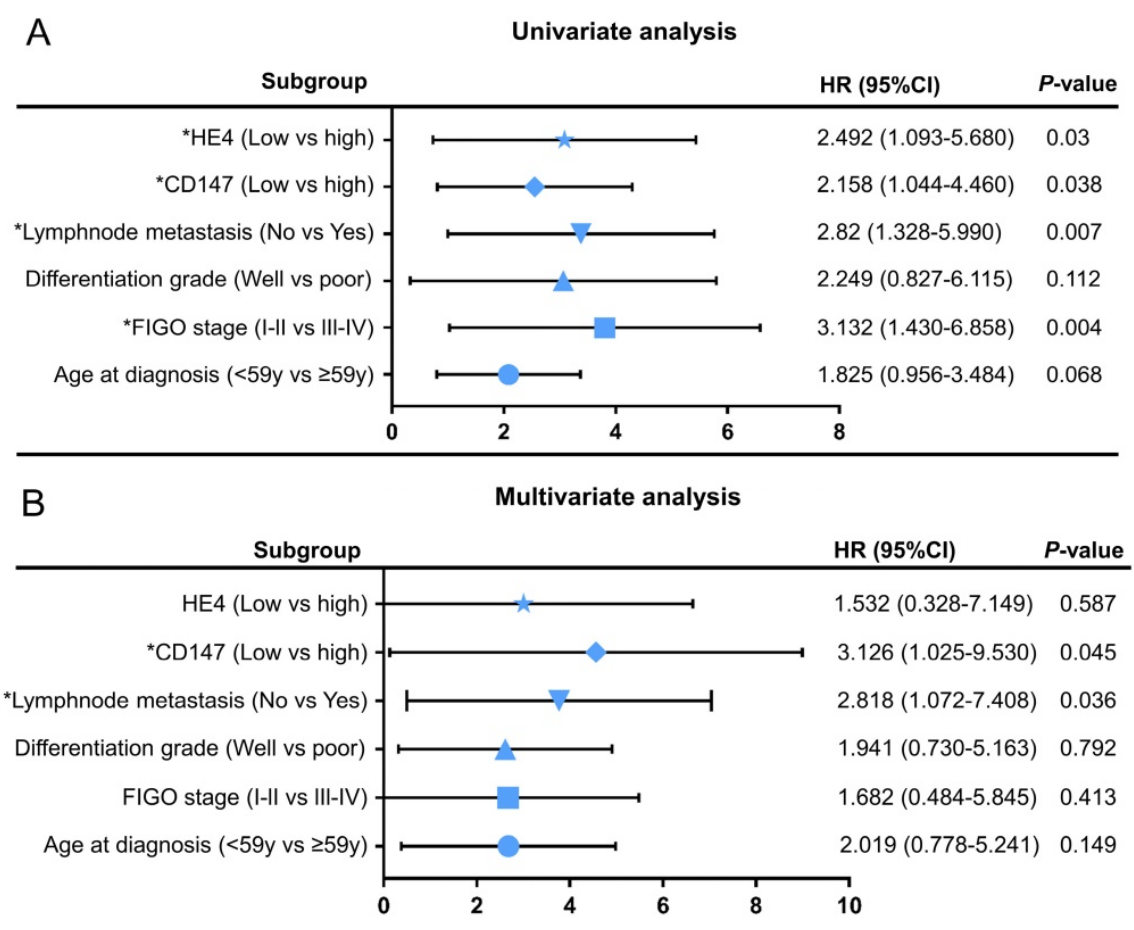

Figure 6. Univariate (A) and multivariate (B) Cox regression analyses by forest map. ${ }^{*} P<0.05$

Correlation between the expression of CD147 and HE4 proteins in ovarian cancer tissues

According to the expression of CD147 and HE4 in 96 cases of ovarian cancer, there were $8,1,5$, and 82 patients in the CD147-/HE4-, D147-/HE4+, CD147+/ HE4- and CD147+/HE4+ groups, respectively. Spearman analysis and Regression model showed 
that the expression of CD147 protein was positively correlated with HE4 protein in ovarian cancer (Spearman correlation coefficient $R s=0.708, P=0.000$ ) (Table 3, Supplementary Figure 2). Univariate linear regression indicated that the expression of CD147 and HE4 could interact with each other (both $P<005$ ). As shown in Table 4, the degree of differentiation can significantly affect the expression of CD147, and the advanced stages are important factors affecting the expression of HE4. Multivariate linear regression suggested that HE4 expression score (0-12) was an independent influence factor of CD147 expression, and CD147 expression was also an independent factor of HE4 expression (Table 4).

Table 3. The correlation between the expression of CDI47 and HE4 in ovarian cancer (the Spearman correlation coefficient Rs $=0.708, P=0.0000$ )

\begin{tabular}{llll}
\hline CD147 & HE4 & \multicolumn{2}{c}{ Cases } \\
\cline { 2 - 3 } & Negative & Positive & \\
\hline Negative & 8 & 1 & 9 \\
Positive & 5 & 82 & 87 \\
Cases & 13 & 83 & 96 \\
\hline
\end{tabular}

\section{Interaction between the expression of CD147 and HE4 in ovarian cancer cells}

We further constructed the stable low expression CD147 ovarian cancer cells OV-CD147-L and ES-CD147-L and their negative control groups OV-NC and ES-NC by lentivirus transfection, the results showed that the expression of HE4 decreased and the expression of AXNA2 protein did not change significantly after the expression of CD147 was inhibited in OV-CD147-L and ES-CD147-L cells by Western blot (Figure 7A and Supplementary figure 3A). Furthermore, the expression of HE4 was inhibited by gene transfection and RNA interference, the result indicated that the expression of CD147 protein in high and low glycosylation forms was decreased after inhibition of HE4 expression (Figure 7B). Stable low expression of ANXA2 ovarian cancer cells OV-shA2-1/OV-shA2-2 and ES-shA2-1/ESshA2-2 and their negative control groups OV-NC and ES-NC were constructed by lentivirus, the results showed that after inhibition of ANXA2 expression, there was no significant change in the expression of CD147 protein (Supplementary Figure 3B). These results suggested that the expression of CD147 and HE4 could interact with each other, however, the expression of CD147 and ANXA2 had no significant effect on each other.

Table 4. The linear regression analysis of CD147 and HE4 expression in ovarian cancer

\begin{tabular}{|c|c|c|c|c|c|c|c|c|}
\hline & \multicolumn{4}{|c|}{ CD147 score } & \multicolumn{4}{|c|}{ HE4 score } \\
\hline & \multicolumn{2}{|c|}{ univariate } & \multicolumn{2}{|c|}{ multivariate } & \multicolumn{2}{|c|}{ univariate } & \multicolumn{2}{|c|}{ multivariate } \\
\hline & $\beta$ & $P$ & $\beta$ & $P$ & $\beta$ & $P$ & $\beta$ & $P$ \\
\hline HE4 score & 0.342 & 0.000 & 0.31 & $0.001^{\#}$ & & & & \\
\hline CD147score & & & & & 0.417 & 0.000 & 0.396 & $0.000^{*}$ \\
\hline Age at diagnosis & 0.011 & 0.718 & & & 0.007 & 0.832 & & \\
\hline FIGO stage & 0.44 & 0.224 & & & 1.167 & 0.003 & & \\
\hline $\begin{array}{l}\text { Differentiation } \\
\text { grade }\end{array}$ & 1.203 & 0.014 & & & 0.997 & 0.068 & & \\
\hline $\begin{array}{l}\text { Lymphnode } \\
\text { metastasis }\end{array}$ & 0.773 & 0.381 & & & 0.489 & 0.618 & & \\
\hline
\end{tabular}

\#Represents the multivariate regression analysis when CD147 score was dependent variate, including differentiation and HE4 score as independent variables;

*represents the multivariate regression analysis when HE4 score was dependent variate, including FIGO stages and CD147 score as independent variables.
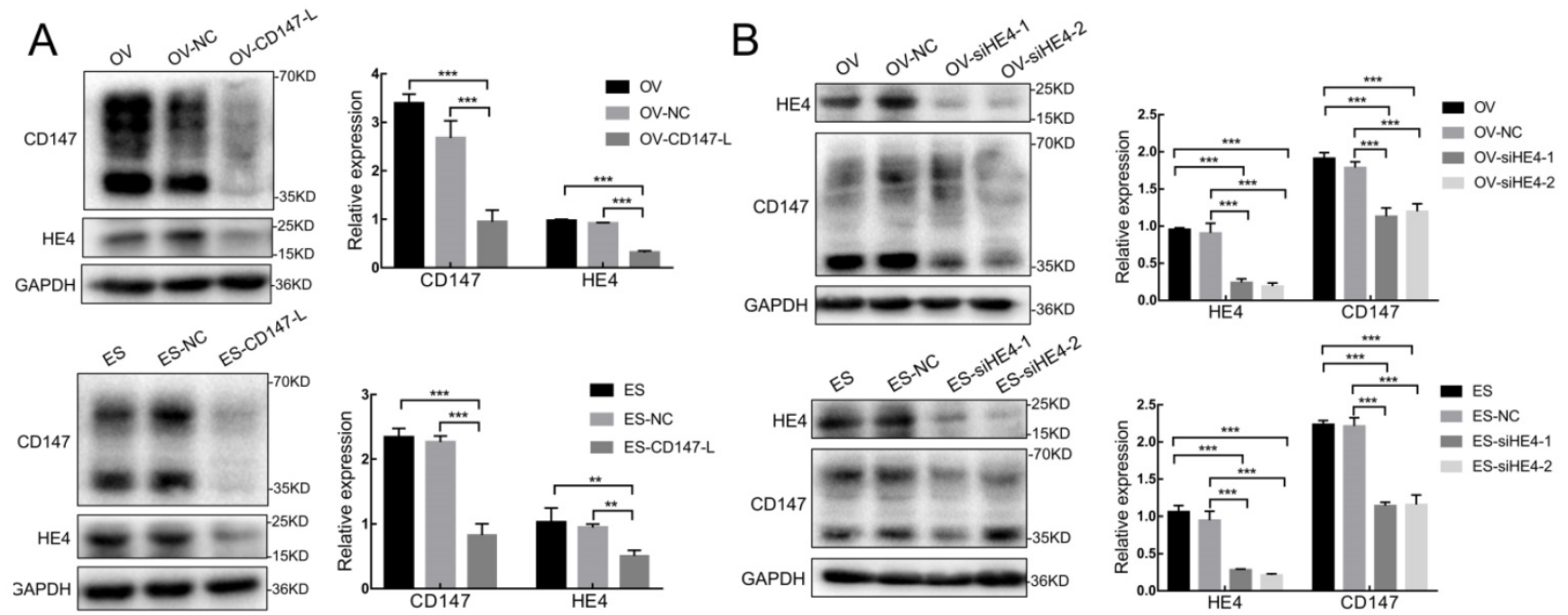

Figure 7. Interaction between CD147 and HE4 expression. (A) The expression of HE4 protein decreased after downregulation of CDI47 protein in OVCAR3 and ES-2 cells by Western blot. (B) The expression of CD147 was downregulated after inhibition of HE4 protein in OVCAR3 and ES-2 cells by Western blot. OV: OVCAR3, ES: ES-2, HE4: human epididymis protein 4, OV-NC: negative control group of OVCAR3 cells, ES-NC: negative control of ES-2 cells, OV-CD147-L: downregulation of CD147 expression in OVCAR3 constructed by lentivirus, ES-2-CD147-L: downregulation of CD147 expression in ES-2 constructed by lentivirus, OV-siHE4-L-1/2: downregulation of HE4 expression in OVCAR3 by siRNA, ES-siHE4-L-1/2: inhibition of HE4 expression in ES-2 by siRNA. 

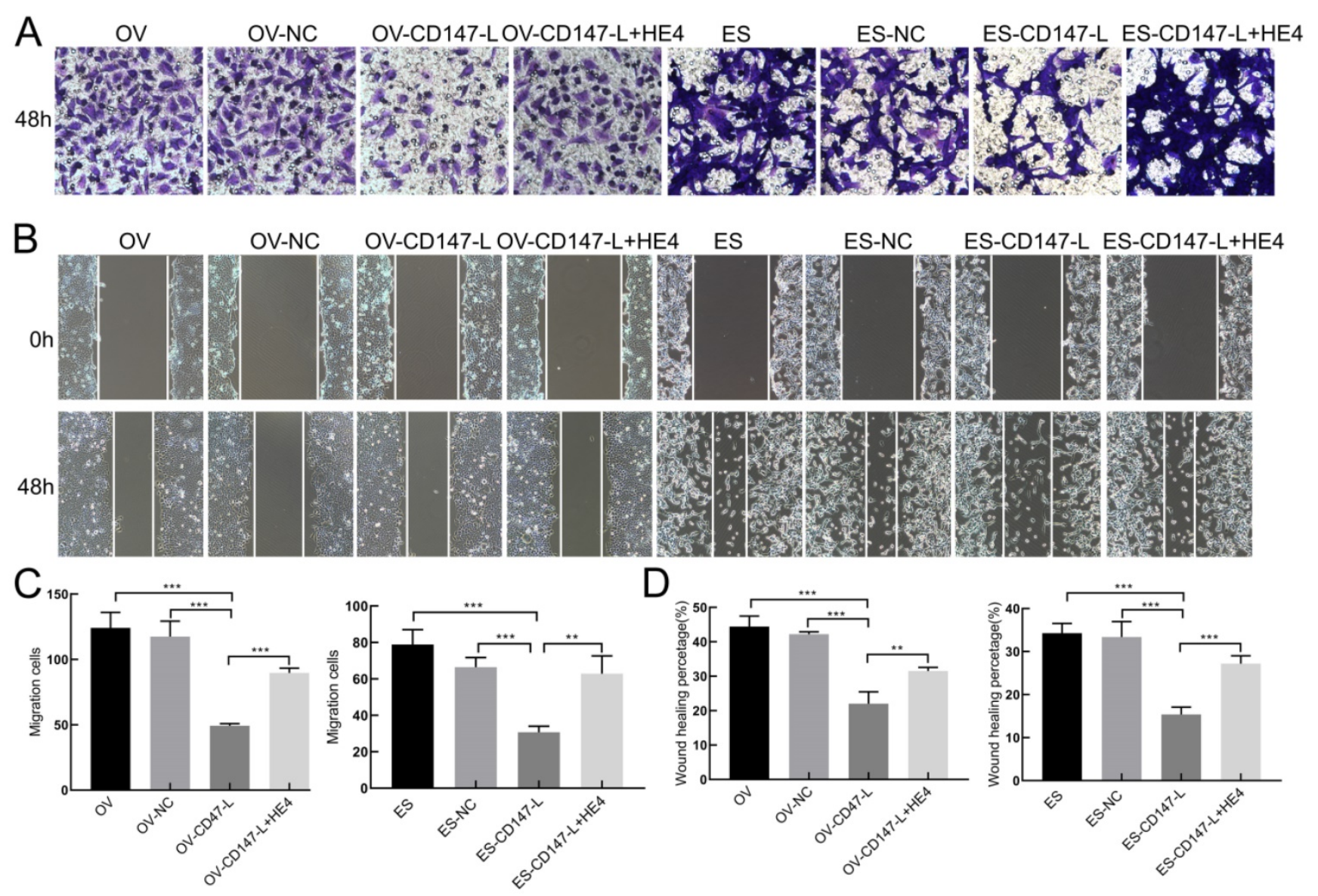

Figure 8. Interaction of CD147 and HE4 promotes invasion and migration of ovarian cancer cells. (A, C) The invasion capacities of ovarian cancer cells (OVCAR3 and ES-2) after downregulation of CD147 protein and addition of recombinant HE4 active protein detected by Transwell assay. (B, D) The migration capacities of ovarian cancer cells (OVCAR3 and ES-2) after downregulation of CD147 protein and addition of recombinant HE4 active protein detected by Scratch test. HE4: human epididymis protein 4.

\section{Interaction between CD147 and HE4 promotes invasion and metastasis of ovarian cancer}

In order to further detect the role of CD147 and HE4 interaction in the invasion and metastasis of ovarian cancer cells, Transwell assay and Scratch test were performed. The results showed that the invasive and migration abilities both decreased after downregulation of CD147 protein in OVACR3 and ES-2 cells, and recovered after the addition of human recombinant HE4 active protein by Transwell assay and Scratch test (Figure 8). These results suggest that HE4 may affect the invasion and migration of ovarian cancer cells by regulating the expression of CD147.

\section{Discussion}

The progression and development of malignant tumor is a multi-factor, multi-step cascade reaction process, including infiltration of primary tumor, degradation of basement membrane, invasion of tumor cells and distant metastasis. Degradation of extracellular matrix is the premise of tumor cell invasion and metastasis, as an extracellular matrix metalloproteinase inducer, CD147 is a cancer-specific biomarker, which can not only mediate intercellular adhesion, but also induce production of extracellular MMPs, playing a key role in promoting tumor invasion and metastasis [23-24]. Researchers found that CD147 protein was up-regulated in many kinds of tumors, such as endometrial carcinoma, colon cancer, liver cancer and lung cancer, regulating malignant progression, infiltration, and invasion of the tumors [25-26, 8, 12]. Studies have shown that CD147 can regulate cytoskeletal movement by activating PI3K/Akt and MAPK signaling pathway, induce the production of vascular endothelial growth factor (VEGF), and enhance anoikis resistance [27-29]. Several investigations demonstrated that CD147 can facilitate the glycolysis of tumor cells in anoxic microenvironment, enhance tumor proliferation, invasion, and inhibit apoptosis under hypoxic condition [30-31]. At present, anti-CD147 monoclonal antibody can inhibit the invasion ability of liver cancer by reducing the expression of MMP2 and MMP9. What's more, anti-CD147 monoclonal antibody can inhibit the occurrence and development of pancreatic cancer, suggesting that anti-CD147 
monoclonal antibody has potential clinical value in drug-resistant recurrence of pancreatic cancer [32].

As a new generation tumor marker of ovarian cancer, HE4 is an exocrine protein encoded by WFDC2 (WAP four-disulfide core protein 2) gene [33]. At present, the research on HE4 is mainly focused on ovarian cancer and endometrial cancer, the level of HE4 gene in ovarian serous carcinoma is significantly higher than that in other cancers, the levels of HE4 gene expression are moderate in transitional cell carcinoma, breast cancer, renal cell carcinoma and pancreatic cancer. However, the expression of HE4 gene was very low in colon cancer, gastric cancer, liver cancer and prostate cancer [34], which may be associated with different genetic backgrounds in various types of tumors. In 2013, Kong et al found that HE4 could inhibit the proliferation abilities of ovarian cancer cells through MAPK and PI3K/AKT signaling pathway, but had no effect on EGFR phosphorylation [35]. Zhuang et al showed that Lewis y fucosylation of HE4 protein promoted the proliferation, invasion and metastasis of ovarian cancer cells [36]. HE4 protein is also significantly increased in endometrial carcinoma, which is helpful for the early diagnosis and treatment of endometrial carcinoma [16]. Furthermore, the high expression of HE4 is associated with lymph node metastasis and shortened disease-free survival in breast cancer, suggesting that HE4 can be used as a prognostic factor for breast cancer [37]. The above studies have suggested that HE4 plays a vital role in the occurrence, development, and biological function of malignant tumors.

In order to investigate the expression and mutual interaction of CD147 and HE4 in ovarian cancer, we confirmed that these two proteins co-precipitated and co-located in ovarian cancer cells by Western blot, immunoprecipitation, and double-labeling immunofluorescence assays. We further confirmed that both CD147 and HE4 proteins were highly expressed in ovarian cancer tissues, and there was a positive correlation between the expression of CD147 and HE4 protein, suggesting that the interaction between CD147 and HE4 plays a crucial part in the occurrence and development of ovarian cancer. Furthermore, high expression of CD147 and HE4 was significantly associated with the differentiation degree and FIGO (III-IV) stages of ovarian cancer, and predicted poor prognosis of ovarian cancer, indicating that the interaction between the two molecules participated in promoting the invasion and metastasis of ovarian cancer and affecting the prognosis of patients with ovarian cancer. Taken together, this study may provide basis therapy for further exploring the interaction of CD147 and HE4 in other malignant tumors.

Most proteins usually form molecular complexes together performing their biological functions in the process of invasion and metastasis of malignant tumors. At present, numerous studies have shown that CD147 can interact with varieties of molecules to regulate the occurrence, development, invasion, and metastasis of tumor, including Annexin A2 (ANXA2), VEGFR-2, MCT1, integrin- $\beta 1$, CD44, cyclophile. Zhao et al. [22] showed that CD147 interacted and co-precipitated with ANXA2 in hepatocellular carcinoma cells, and could promote the movement and invasion of tumor cells. CD147 also regulated the activation of VEGFR-2 and downstream signaling pathway by directly binding to VEGFR-2 [38]. Walters et al [39] showed the interaction of CD147 with MCT1 and MCT4 promoted glycolysis and accelerated the malignant progression of tumors. Additionally, the interaction between CD147 and integrin- $\beta 1$ promoted the invasion and metastasis of hepatocellular carcinoma [40]. In melanoma cells, CD147 interacted with cyclophile to regulate $\mathrm{Ca}^{+}$channel and MMPs activities [41].

At present, studies showed that HE4 can interact with HIF-a, EGFR, IGF1R, ANXA2 and other protein molecules. Researchers found that the expression of HE4 decreased after inhibition of HIF1-a expression or treated with HIF1-a inhibitor in tumor cells; HE4 could co-immunoprecipitate and co-locate with EGFR, and the interaction between HE4 and EGFR was enhanced after upregulation of HE4 expression [42]. Our study suggested that the invasion and migration abilities were both inhibited after downregulation of CD147 protein and recovered after the addition of human recombinant HE4 active protein, indicating that CD147 and HE4 could interacted with each other to form a protein complex, and HE4 could promote invasion and metastasis of ovarian cancer by regulating the expression of CD147. We further confirmed that ANXA2, as another CD147-interacting protein in ovarian cancer, had no effect on the expression of CD147. Previous studies have found that the interaction between ANXA2 and HE4 promotes the invasion and migration of ovarian cancer cells by activating MAPK and FOCAL signaling pathways $[16,20]$. Moreover, HE4-AXNA2MMP2 can form protein complex to promote the invasion and metastasis of malignancy [21]. Therefore, we speculate that the interaction between CD147 and HE4 may regulate the occurrence, development, invasion and metastasis of ovarian cancer in the form of protein complex, in which ANXA2 may play a "bridge" role. In the follow-up experiments, we will continue to explore the effect and underlying mechanism of CD147-HE4-ANXA2 
protein complex on the invasion and metastasis of ovarian cancer.

In conclusion, this study confirmed for the first time that CD147 and HE4 protein could interact with each other and were both associated with the poor prognosis of ovarian cancer, and HE4 can promote the invasion and metastasis of ovarian cancer by regulating the expression of CD147, ANXA2 may play a "bridge" role in this process, which provides a theoretical basis for the further study of underlying mechanism of CD147 and HE4 interaction in ovarian cancer.

\section{Abbreviations}

OC: ovarian cancer; HE4: human epididymis protein 4; WFDC2: WAP four-disulfide core protein 2; ANXA2: Annexin2; EMMPRIN: extracellular matrix metalloproteinase inducer; IHC: Immunohistochemistry; ICC: Immunocytochemistry; MMP: matrix metalloprotinase; VEGF: vascular endothelial growth factor; MAPK: mitogen-activated protein kinase; PI3K/AKT: phosphatidylinositol 3-kinase pathway/ Protein Kinase B.

\section{Supplementary Material}

Supplementary figures and tables. https://www.jcancer.org/v12p7422s1.pdf

\section{Acknowledgements}

This work was supported by grants from the National Natural Science Foundation of China (No. 82173130, No.81472437 and No. 81672590) and Outstanding Scientific Fund of Shengjing Hospital (No. 201804), the Key R\&D Guidance Plan Project in Liaoning Province (2019JH8/10300022), Beijing Kanghua Foundation for the Development of Traditional Chinese and Western Medicine Gynecological Oncology Special Research Fund (KH-2021-LLZX-010).

\section{Availability of data and materials}

The datasets used or analyzed during the current study are available from the corresponding author upon reasonable request.

\section{Ethics approval and patients' consent for publication}

We have obtained the approval from the Institution's Ethics Committee of Shengjing hospital of China Medical University. The approval number is 2021PS388K(X1). This study did not contain any work with animals performed by any of the authors. Therefore, local ethics approval about animals was not needed.

\section{Authorship and contributions}

Lingling Gao and Xin Nie collected and present the data. Lingling Gao and Rui Gou performed the experiments and wrote the manuscript, Lingling Gao, Yue Qi and Juanjuan Liu performed the analysis and functional experiments. Juanjuan Liu and Bei Lin designed the studies and revised the manuscript. All authors have read and approved the manuscript.

\section{Competing Interests}

The authors have declared that no competing interest exists.

\section{References}

1. Moufarrij S, Dandapani M, Arthofer E, et al. Epigenetic therapy for ovarian cancer: promise and progress. Clin Epigenetics. 2019;11(1):7

2. Cortez AJ, Tudrej P, Kujawa KA, et al. Advances in ovarian cancer therapy. Cancer Chemother Pharmacol. 2018;81(1):17-38.

3. Suh DH, Kim M, Lee KH, et al. Major clinical research advances in gynecologic cancer in 2017. J Gynecol Oncol. 2018;29(2): e31.

4. Miyauchi T, Kanekura T, Yamaoka A, et al. Basigin, a new, broadly distributed member of the immunoglobulin superfamily, has strong homology with both the immunoglobulin $\mathrm{V}$ domain and the beta-chain of major histocompatibility complex class II antigen. J Biochem. 1990;107(2):316-23.

5. Biswas $\mathrm{C}$, Zhang $\mathrm{Y}$, DeCastro $\mathrm{R}$, et al. The human tumor cell-derived collagenase stimulatory factor (renamed EMMPRIN) is a member of the immunoglobulin superfamily. Cancer Res. 1995;55(2):434-9.

6. Fossum S, Mallett S, Barclay AN. The MRC OX-47 antigen is a member of the immunoglobulin superfamily with an unusual transmembrane sequence. Eur J Immunol. 1991;21(3):671-9.

7. Toole BP. Emmprin (CD147), a cell surface regulator of matrix metalloproteinase production and function. Curr Top Dev Biol. 2003; 54:371-89.

8. Liu M, Tsang JYS, Lee M, et al. CD147 expression is associated with poor overall survival in chemotherapy treated triple-negative breast cancer. J Clin Pathol. 2018:71(11):1007-14.

9. Lu M, Wu J, Hao ZW, et al. Basolateral CD147 induces hepatocyte polarity loss by E-cadherin ubiquitination and degradation in hepatocellular carcinoma progress. Hepatology. 2018;68(1):317-32.

10. Shou ZX, Jin X, Zhao ZS. Upregulated expression of ADAM17 is a prognostic marker for patients with gastric cancer. Ann Surg. 2012;256(6):1014-22.

11. Zhou Y, Wu B, Li JH, et al. Rab22a enhances CD147 recycling and is required for lung cancer cell migration and invasion. Exp Cell Res. 2017;357(1):9-16.

12. Liu J, Liu Q, Wang Y, et al. Coexpression of Lewis y antigen and CD147 in epithelial ovarian cancer is correlated with malignant progression and poor prognosis. Int J Mol Med. 2019;43(4):1687-98.

13. Hellstrom I, Raycraft J, Hayden-Ledbetter M, et al. The HE4 (WFDC2) protein is a biomarker for ovarian carcinoma. Cancer Res. 2003;63(13):3695-700.

14. Moore RG, Brown AK, Miller MC, et al. The use of multiple novel tumor biomarkers for the detection of ovarian carcinoma in patients with a pelvic mass. Gynecol Oncol. 2008;108(2):402-8.

15. Moore RG, McMeekin DS, Brown AK, et al. A novel multiple marker bioassay utilizing HE4 and CA125 for the prediction of ovarian cancer in patients with a pelvic mass. Gynecol Oncol. 2009;112(1):40-6

16. Deng L, Gao Y, Li X, et al. Expression and clinical significance of annexin A2 and human epididymis protein 4 in endometrial carcinoma. J Exp Clin Cancer Res. 2015;34:96.

17. Liu W, Yang J, Chi PD, et al. Evaluating the clinical significance of serum HE4 levels in lung cancer and pulmonary tuberculosis. Int J Tuberc Lung Dis. 2013;17(10):1346-53.

18. Kamei M, Yamashita S, Tokuishi K, et al. HE4 expression can be associated with lymph node metastases and disease-free survival in breast cancer. Anticancer Res. 2010:30(11):4779-83.

19. O'Neal RL, Nam KT, LaFleur BJ, et al. Human epididymis protein 4 is up-regulated in gastric and pancreatic adenocarcinomas. Hum Pathol. 2013;44(5):734-42.

20. Zhuang H, Tan M, Liu J, et al. Human epididymis protein 4 in association with Annexin II promotes invasion and metastasis of ovarian cancer cells. Mol Cancer. 2014; 13:243.

21. Wang J, Deng $\mathrm{L}$, Zhuang $\mathrm{H}$, et al. Interaction of HE4 and ANXA2 exists in various malignant cells-HE4-ANXA2-MMP2 protein complex promotes cell migration. Cancer Cell Int. 2019; 19:161.

22. Zhao P, Zhang W, Tang J, et al. Annexin II promotes invasion and migration of human hepatocellular carcinoma cells in vitro via its interaction with HAb18G/CD147. Cancer Sci. 2010;101(2):387-95.

23. Huang Y, Jiang J, Dou K, et al. HAb18G/CD147 enhances the secretion of matrix metalloproteinases (MMP) via cGMP/NO-sensitive capacitative 
calcium entry (CCE) and accordingly attenuates adhesion ability of fibroblasts. Eur J Cell Biol. 2005;84(1):59-73.

24. Zhou J, Zhu P, Jiang JL, et al. Involvement of CD147 in overexpression of MMP-2 and MMP-9 and enhancement of invasive potential of PMA-differentiated THP-1. BMC Cell Biol. 2005;6(1):25.

25. Gao J, Hu Z, Liu J, et al. Expression of CD147 and Lewis y antigen in ovarian cancer and their relationship to drug resistance. Med Oncol. 2014;31(5):920.

26. Hu $\mathrm{XX}, \mathrm{Xu} \mathrm{XN}, \mathrm{He}$ BS, et al. microRNA-485-5p Functions as a Tumor Suppressor in Colorectal Cancer Cells by Targeting CD147. J Cancer. 2018;9(15):2603-11.

27. Dai L, Bratoeva M, Toole BP, et al. KSHV activation of VEGF secretion and invasion for endothelial cells is mediated through viral upregulation of emmprin-induced signal transduction. Int J Cancer. 2012;131(4):834-43.

28. Yang JM, $\mathrm{O}^{\prime} \mathrm{Neill} \mathrm{P}$, Jin $\mathrm{W}$, et al. Extracellular matrix metalloproteinase inducer (CD147) confers resistance of breast cancer cells to Anoikis through inhibition of Bim. J Biol Chem. 2006;281(14):9719-27.

29. Ke X, Li L, Dong HL, et al. Acquisition of anoikis resistance through CD147 upregulation: A new mechanism underlying metastasis of hepatocellular carcinoma cells. Oncol Lett. 2012;3(6):1249-54.

30. Ke X, Chen Y, Wang P, et al. Upregulation of CD147 protects hepatocellular carcinoma cell from apoptosis through glycolytic switch via HIF-1 and MCT-4 under hypoxia. Hepatol Int. 2014;8(3):405-14.

31. Ke X, Fei F, Chen Y, et al. Hypoxia upregulates CD147 through a combined effect of HIF-1alpha and Sp1 to promote glycolysis and tumor progression in epithelial solid tumors. Carcinogenesis. 2012;33(8):1598-607.

32. Fan $X Y, H e D$, Sheng CB, et al. Therapeutic anti-CD147 antibody sensitizes cells to chemoradiotherapy via targeting pancreatic cancer stem cells. Am J Transl Res. 2019;11(6):3543-54.

33. Kirchhoff C, Habben I, Ivell R, et al. A major human epididymis-specific cDNA encodes a protein with sequence homology to extracellular proteinase inhibitors. Biol Reprod. 1991;45(2):350-7.

34. Galgano MT, Hampton GM, Frierson HF, Jr. Comprehensive analysis of HE4 expression in normal and malignant human tissues. Mod Pathol. 2006;19(6):847-53.

35. Kong $\mathrm{X}$, Chang $\mathrm{X}$, Cheng $\mathrm{H}$, et al. Human epididymis protein 4 inhibits proliferation of human ovarian cancer cells via the mitogen-activated protein kinase and phosphoinositide 3-kinase/AKT pathways. Int J Gynecol Cancer. 2014;24(3):427-36

36. Zhuang $\mathrm{H}, \mathrm{Hu} \mathrm{Z}$, Tan $\mathrm{M}$, et al. Overexpression of Lewis y antigen promotes human epididymis protein 4-mediated invasion and metastasis of ovarian cancer cells. Biochimie. 2014;105:91-8.

37. Lu M, Ju S, Shen X, et al. Combined detection of plasma miR-127-3p and HE4 improves the diagnostic efficacy of breast cancer. Cancer Biomark. 2017;18(2):143-8.

38. Bougatef F, Quemener C, Kellouche S, et al. EMMPRIN promotes angiogenesis through hypoxia-inducible factor-2alpha-mediated regulation of soluble VEGF isoforms and their receptor VEGFR-2. Blood. 2009;114(27):5547-56.

39. Walters DK, Arendt BK, Jelinek DF. CD147 regulates the expression of MCT1 and lactate export in multiple myeloma cells. Cell Cycle. 2013;12(19):3175-83.

40. Cui J, Huang W, Wu B, et al. N-glycosylation by N-acetylglucosaminyltransferase V enhances the interaction of CD147/basigin with integrin beta1 and promotes HCC metastasis. J Pathol. 2018;245(1):41-52.

41. Long T, Su J, Tang W, et al. A novel interaction between calcium-modulating cyclophilin ligand and Basigin regulates calcium signaling and matrix metalloproteinase activities in human melanoma cells. Cancer Lett. 2013;339(1):93-101.

42. Moore RG, Hill EK, Horan T, et al. HE4 (WFDC2) gene overexpression promotes ovarian tumor growth. Sci Rep. 2014; 4:3574. 